\title{
Perfil epidemiológico de pacientes Chagásicos Agudos registrados no sistema de notificações e agravos (SINAN) do Estado do Tocantins no período de 2007 a 2018
}

\section{Epidemiological profile of Acute Chagasic patients registered in the system of notifications and injuries (SINAN) of the State of Tocantins in the period from 2007 to 2018}

Diego Santos Andrade1, Brenda Pereira Teles², Daiene Isabel da Silva Lopes³, Durval Nolasco das Neves Neto 4 .

\section{RESUMO}

A doença de Chagas é uma patologia infecciosa que pode ocorrer de forma aguda ou crônica e é causada pelo protozoário Trypanosoma cruzi, podendo ser transmitida pelas vias oral, vetorial, vertical, entre outras No Tocantins o consumo de alimentos naturais típicos da região como bacaba, açaí e palmito de babaçu predispõe a população tocantinense ao contato com o protozoário e consequentemente a infecção pela Doença de Chagas na forma aguda. O objetivo deste trabalho foi de levantar, descrever e apresentar o perfil epidemiológico dos pacientes chagásicos agudos no Estado do Tocantins no período de 2007 e 2018. Foram coletados os dados de todos os casos de Doença de Chagas Aguda (DCA) confirmados no Estado do Tocantins, entre 2007 e 2018, e que foram notificados no Sistema de Informações e Agravos de Notificações (SINAN). No período analisado foram registrados 48 casos de Doença de Chagas Aguda no Estado do Tocantins, com 83,34\% dos casos ocorrendo no segundo semestre dos anos analisados, 56,25\% em mulheres, a faixa etária mais acometida 15 aos 39 anos, $68,75 \%$ pessoa pardas e em $91,66 \%$ dos casos a escolaridade não foi informada na notificação, $79,16 \%$ moradores da zona urbana. Dessa forma concluímos que o perfil epidemiológico dos pacientes estudados é um indivíduo adulto, do sexo feminino, raça parda, escolaridade não informada em grande parte das notificações, morador da zona urbana, com infecção de modo oral e em domicílio.

Palavras-chave: Doença de Chagas; epidemiologia; perfil de saúde; Trypanosoma
${ }^{1}$ Acadêmico UNITPAC.

E-mail: diego-034@live.com

${ }^{2}$ Acadêmica de enfermagem. UNITPAC.

Doutora em ciência animal tropical. UNITPAC.

${ }^{4}$ Doutor em ciência animal tropical. UNITPAC.

\section{ABSTRACT}

Chagas' disease is an infectious pathology that can occur in an acute or chronic form and is caused by the protozoan Trypanosoma cruzi, which can be transmitted by oral, vector, vertical routes, among others In Tocantins the consumption of natural foods typical of the region such as bacaba, açaí and babassu palm heart predisposes the Tocantins population to contact with the protozoan and consequently the acute Chagas disease infection. The aim of this study was to survey, describe and present the epidemiological profile of acute chagasic patients in the State of Tocantins in the period 2007 and 2018. Data were collected for all confirmed cases of Acute Chagas Disease (DCA) in the State of Tocantins, between 2007 and 2018, and which were notified in the Information System and Notifiable Diseases (SINAN). In the analyzed period, 48 cases of Acute Chagas Disease were recorded in the State of Tocantins, with 83.34\% of cases occurring in the second half of the years analyzed, $56.25 \%$ in women, the most affected age group 15 to 39 years, 68 , 75\% brown people and in $91.66 \%$ of cases the education was not informed in the notification, $79.16 \%$ lived in the urban area. Thus, we conclude that the epidemiological profile of the patients studied is an adult, female, brown race, education level not informed in most of the notifications, living in the urban area, with oral infection and at home.

Keywords: Acute Chagas disease. Trypanosoma. Barber. 


\section{INTRODUÇAOO}

A doença de Chagas aguda (DCA) é uma doença provocada pelo Tripanosoma cruzi, e tem como vetor o Triatoma Infestans que é um triatomíneo hematófago (RIBEIRO et al., 2017). Diversos são os meios de transmissibilidade dessa doença, como por exemplo a via oral, vetorial, vertical, acidental, transfusão sanguínea e até mesmo transplante de órgãos de pessoas contaminadas(VARGAS et al., 2018).

A DC tem duas formas de apresentação: Aguda e crônica. Geralmente ela ocorre de forma assintomática ou, se os sinais e sintomas estiverem presentes, eles ocorrem de forma pouco evidente como por exemplo astenia, cefaleia, febre (LOYANE; CARVALHO, 2018). Quando a febre se apresenta de forma intensa junto com adenomegalia e hepatoesplenomegalia tem-se caracterizado uma forma grave da DCA que provável evolução para óbito (SANTOS; PEREIRA, 2013).

Dessa forma, como a DCA pode ocorrer de forma assintomática, em alguns casos pode passar desapercebida e somente ser diagnosticada na forma crônica quando se apresenta com alterações estruturais nos órgãos, principalmente no coração. Analisando por esse ângulo pode-se entender o fator de apenas uma pequena quantidade de casos de DCA são notificados (WANDERLEY et al., 2007).

Para realização do diagnóstico da DCA utiliza-se a pesquisa do protozoário na forma tripomastigotas em amostras de sangue do paciente (TATTO; PADILHA; FERNANDES, 2007). Pode-se encontrar uma quantidade elevada de tripomastigotas na fase aguda, caracterizando a parasitemia. Os exames utilizados são parasitológicos direto, a fresco, gota espessa entre outros (COSTA et al., 2013).

A Organização Mundial da Saúde (OMS) considera a doença de Chagas como uma doença negligenciada. As patologias negligenciadas são aquelas provocadas por agentes infecciosos ou parasitas e consideradas endêmicas em populações de baixa renda. Essas doenças possuem elevados indicadores de acometimento populacional e pouquíssimo investimento em pesquisas, produção de medicamentos e em seu controle (SANTOS; PEREIRA, 2014). A OMS estima que 6 milhões de pessoas estejam infectadas em 21 países da América Latina, de 7 a 8 milhões no mundo e calcula-se que 70 milhões de pessoas estejam em risco de contrair a doença. Calcula-se que causa 14.000 mortes por ano na região (FERREIRA; BRANQUINHO; LEITE, 2014).

No período de 2010 a 2019, foram notificados 2612 casos confirmados de DCA na maioria dos estados brasileiros. Desses casos pôde-se verificar que 2457 (94\%) estava 
concentrado na região Norte. E quando se analisa as principais formas de transmissão relacionadas a esses casos, 76\% foram por transmissão oral, 8\% por transmissão vetorial e em 15\% não foi identificada a forma de transmissão (FLÁVIA et al., 2020).

Analisando a questão da cronicidade versus forma aguda, pode-se observar a avassaladora desproporção existente entre o número de casos de DCA e os crônicos. São cerca de 500 mil pacientes com a forma crônica no Brasil, e o número casos agudos diagnosticados é extremamente inferior (RASSI; RASSI, 2010).

Existe a predominância, no Brasil, dos pacientes na forma crônica da doença de Chagas e a grande maioria teve a infecção pelo modo vetorial (SANGENIS et al., 2017). Hoje em dia houve uma virada na forma de infecção e tem-se como principal meio a infecção oral, através do consumo de alimentos como açaí ou frutas consumidas in natura (PINTO et al., 2010). Analisando o perfil epidemiológico de acometimento observa-se uma grande quantidade de casos por transmissão oral no Brasil, principalmente na região Amazônica (SHAW; LAINSON; FRAIHA, 1969).

No Tocantins por pertencer a região Amazônica, os casos diagnosticados têm uma expressão muito alta, o que reforça ainda mais permanente vigilância e estudo das variáveis envolvidas na infecção dos pacientes deste estado. Essas medidas são necessárias para um correto controle do agravo, e para que se tenha êxito na intervenção e elaboração de medidas preventivas para diminuir a incidência da doença (ORTIZ et al., 2019). Esse estudo epidemiológico tem como principal objetivo oferecer uma descrição do perfil epidemiológico de acometimento dos pacientes diagnosticados com doença de Chagas em fase aguda no Tocantins, em um estudo retrospectivo, entre 2007 e 2018.

Esse tipo de estudo é importante para manter os agentes públicos locais munidos de informações consolidadas que possam nortear a conduta e enfrentamento à Doença de Chagas. É necessário para se manter uma vigilância epidemiológica atenta e, como citado anteriormente, munida de informações que traduzam os acontecimentos do Estado do Tocantins e assim se possa ter uma atuação mais efetiva e direcionada para a realidade local.

\section{MATERIAIS E METODOS}

Trata-se de uma pesquisa de cunho epidemiológico, descritivo e retrospectivo, com abordagem quantitativa. A coleta dos foi realizada de forma eletrônica através do Sistema de Informação de Agravos de Notificação (SINAN). Os dados considerados foram dos 
pacientes diagnosticados com doença de Chagas Aguda no Estado do Tocantins entre 2007 e 2018. As variáveis estudadas foram: Casos confirmados por mês de primeiro sintoma, zona de residência, modo de infecção, faixa etária, escolaridade, raça, sexo.

Os critérios de inclusão foram as notificações de casos novos em pacientes com diagnóstico de doença de Chagas aguda, no estado do Tocantins, registradas no SINAN, no período escolhido. Os critérios de exclusão foram as notificações duvidosas, incompletas, com registro de outro período e as variáveis não selecionadas para este estudo.

Inicialmente foi realizado o processamento dos dados no Microsoft Office Excel e posteriormente foram tratados estatisticamente para obtenção dos gráficos e tabelas. Posteriormente foi realizada uma análise estatística descritiva para obtenção dos resultados e posteriormente interpretação. Por se tratar de estudo através de dados secundários não foi necessário a submissão ao comitê de ética em pesquisa.

\section{RESULTADOS}

Segundo Sangenis et al. (2016), inúmeras mudanças na epidemiologia da Doença de Chagas aconteceram. Muitas delas se devem ao fato de efetivas ações de controle da transmissão na forma vetorial em diversos países. Entretanto no Brasil a prevalência ainda é elevada.

No período estudado a incidência média anual foi de aproximadamente 4 casos de Doença de Chagas Aguda (DCA). A maior incidência desta doença foi no ano de 2011 e 2018 com 16 casos confirmados. Por outro lado, a menor incidência ocorreu no ano de 2007 com apenas 1 caso confirmado.

Os municípios acometidos foram Araguaína, Ananás, Augustinópolis e Palmas. Considerando o local de notificação temos em primeiro lugar Araguaína com 15 casos, seguida por Palmas 12 casos, Ananás com 10, e Augustinópolis com 5, Araguatins e Porto Nacional com 1 caso. Dos 48 casos registrados, 4 ficaram sem ter a classificação de cidade registrada, tendo no total apenas 44 casos classificados de acordo com o município em que foi notificado.

Esse padrão de acometimento pode ser explicado pelo fato das pessoas com DCA migrarem para outros municípios com centros de referência para diagnóstico ou tratamento da doença, local onde é feita a notificação. 


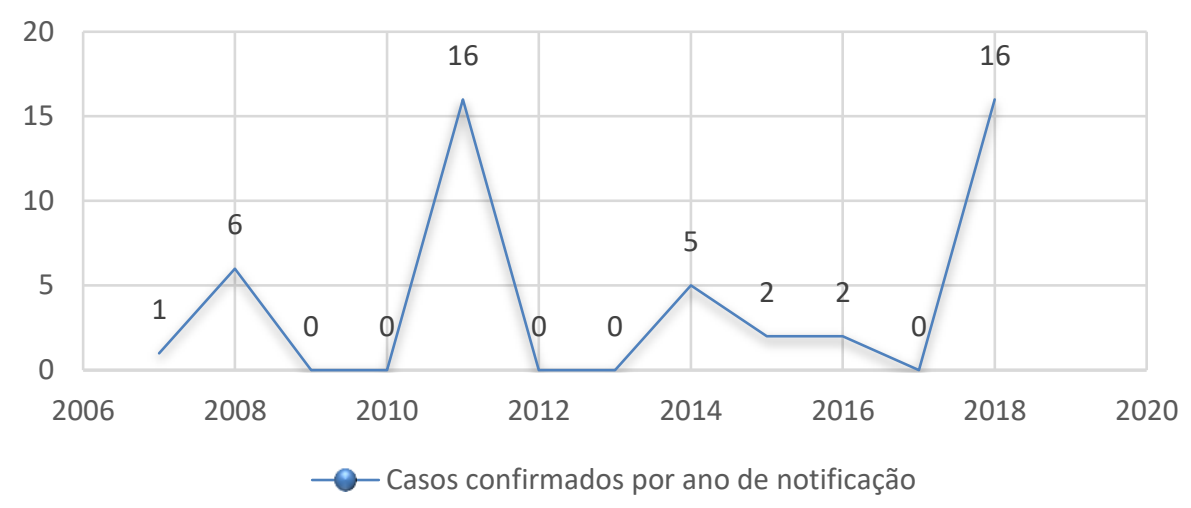

Figura 1: Casos confirmados por ano de notificação.

As safras de açaí acontecem de forma mais abundante no segundo semestre do ano. Isso se deve ao fato desse período ser marcado pela estiagem, contribuindo para uma produção cerca de 2 a 5 vezes maior que no primeiro semestre. Diante desse fator e com aumento da produção, reduz-se o preço e a fruta torna-se mais disponível, aumentando o seu consumo pela população (ALMEIDA et al., 2016).

Quanto a sazonalidade de acontecimento dos dados, foram registrados entre os anos de 2007 e 2018, no estado do Tocantins, 48 casos de doença de Chagas aguda (DCA). Desses casos, pode-se observar que $16,66 \%$ ocorreram no primeiro semestre dos anos analisados e, consequentemente $83,34 \%$ no segundo semestre. Conforme demonstrado na figura 2 observa-se que a maioria dos casos ocorreu no segundo semestre de todos os anos analisados, ressaltando-se os meses de outubro e novembro. Esses meses são os mais secos do ano e são caracterizados por baixos índices pluviométricos e altas temperaturas. Junto com esses fatores temos a ocorrência de queimadas e desflorestamentos o que agrava ainda mais a situação das altas temperaturas.

Dessa maneira, o maior o número de casos de DCA no segundo semestre se deve a temperaturas mais elevadas o que provavelmente provoca a dispersão dos agentes causadores da doença. Dessa maneira, eles se deslocam de seu ambiente silvestre e rumam ao peridomicílio humano o que torna maiores as chances de transmissão vetorial. Outro ponto que vale destacar, como mencionado anteriormente, é que no segundo semestre do ano é o que ocorre as maiores safras de açaí, que, de uma maneira ou de outra poderá ser contaminado pelo agente causador da DCA e acabar por via oral realizando a contaminação dos pacientes. 


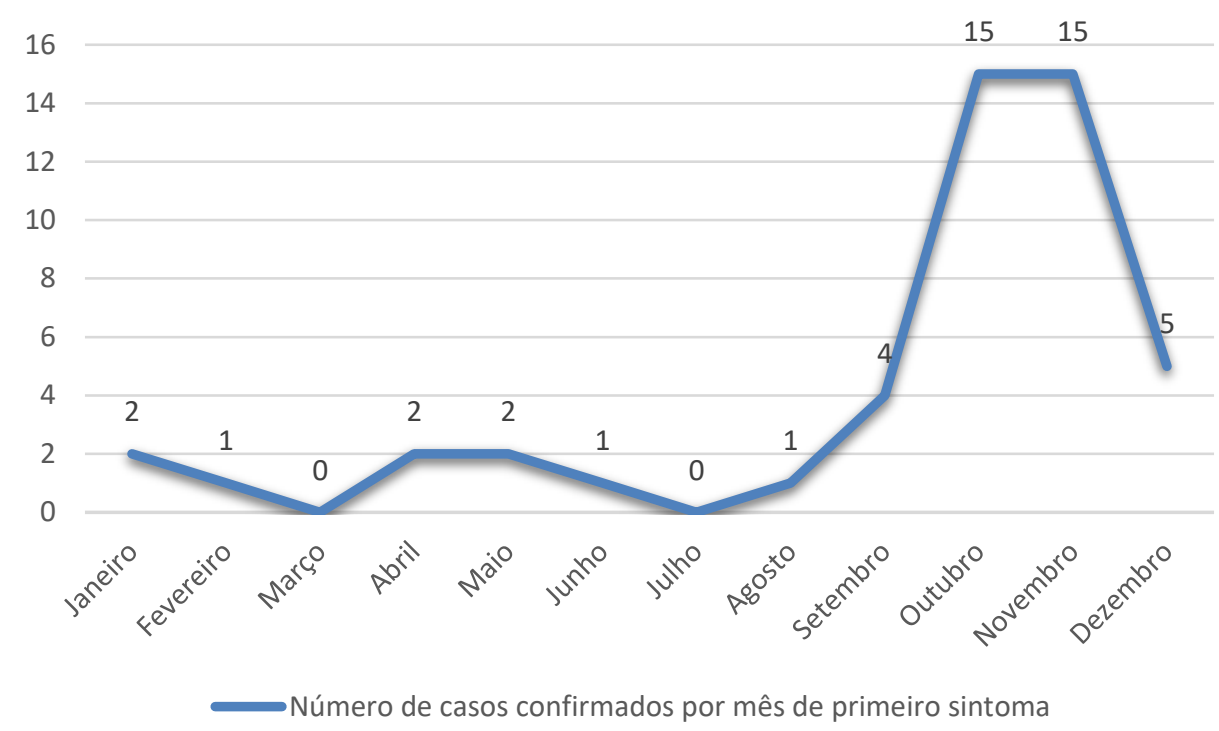

Figura 2: Casos confirmados por mês de primeiro sintoma

Estratificando os casos de DCA por sexo de acometimento, houve um maior acometimento do sexo feminino. Mas essa variável não nos dá a possibilidade de afirmar que existe algum aspecto da fisiopatologia que favoreça esse acontecimento. Ocorreram 21 casos em homens $(43,75 \%)$ e 27 casos em mulheres $(56,25 \%)$ dos casos em mulheres, conforme exposto na figura 3 .

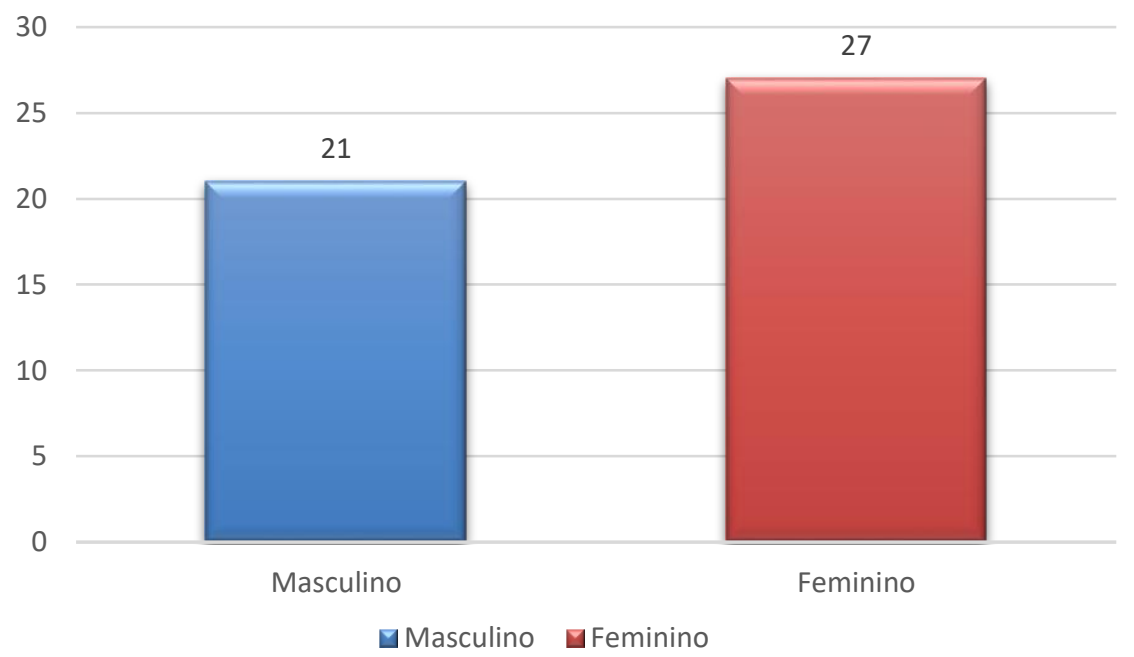

Figura 3: Casos confirmados por sexo.

A faixa etária mais acometida foi entre 15-39 anos, com 15 casos confirmados, seguida pela faixa de 40-59 anos com 9 casos. A menos acometida foi a faixa de 0-04 anos, 
com 3 casos. Analisando esses dados podemos concluir que quanto maior a faixa etária, maior o tempo de exposição e, sendo assim, maiores os riscos de contrair a doença. Esse resultado vai de encontro com os achados da pesquisa de Santos e Pereira (2014) em que a faixa etária mais predominante foi a de 15 aos 59 anos, havendo predomínio do sexo feminino em adultos.

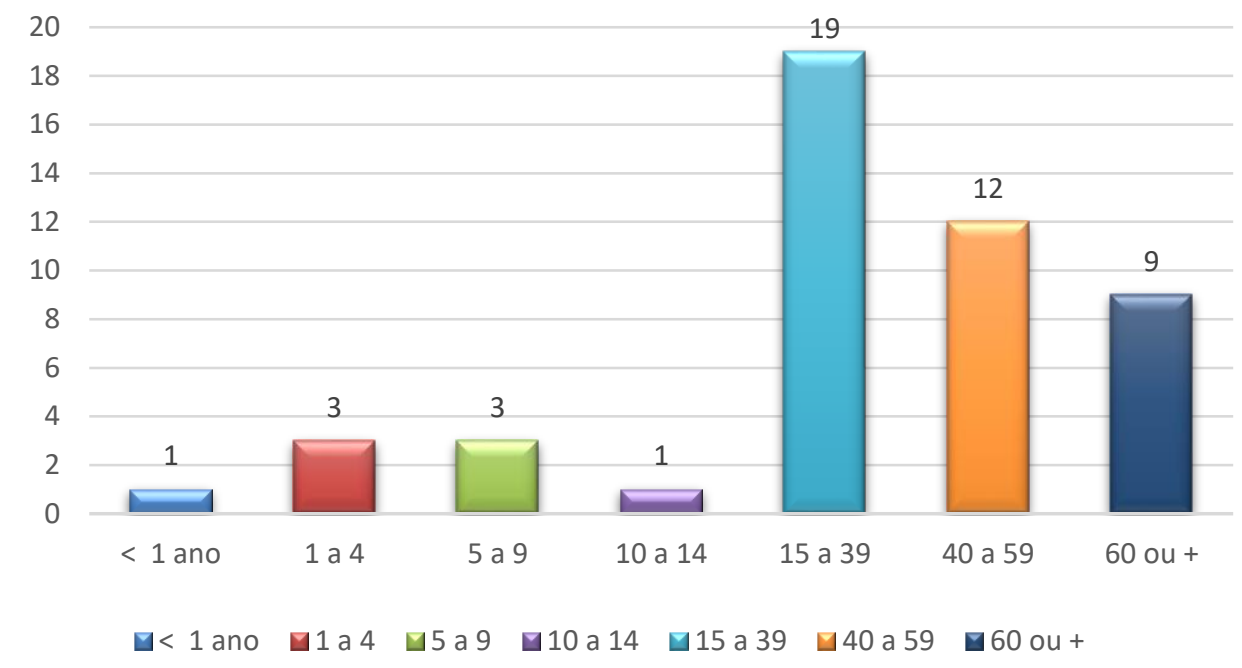

Figura 4: Casos confirmados por faixa etária.

Dentre os indivíduos acometidos pela doença $68,75 \%$ eram da raça parda (figura 5). Esse fato se deve principalmente devido ao Tocantins possuir, segundo o Instituto Brasileiro de Geografia e Estatística, 73,3\% da população parda. 27,08\% ocorreram em pacientes brancos e $4,16 \%$ em pacientes pretos.

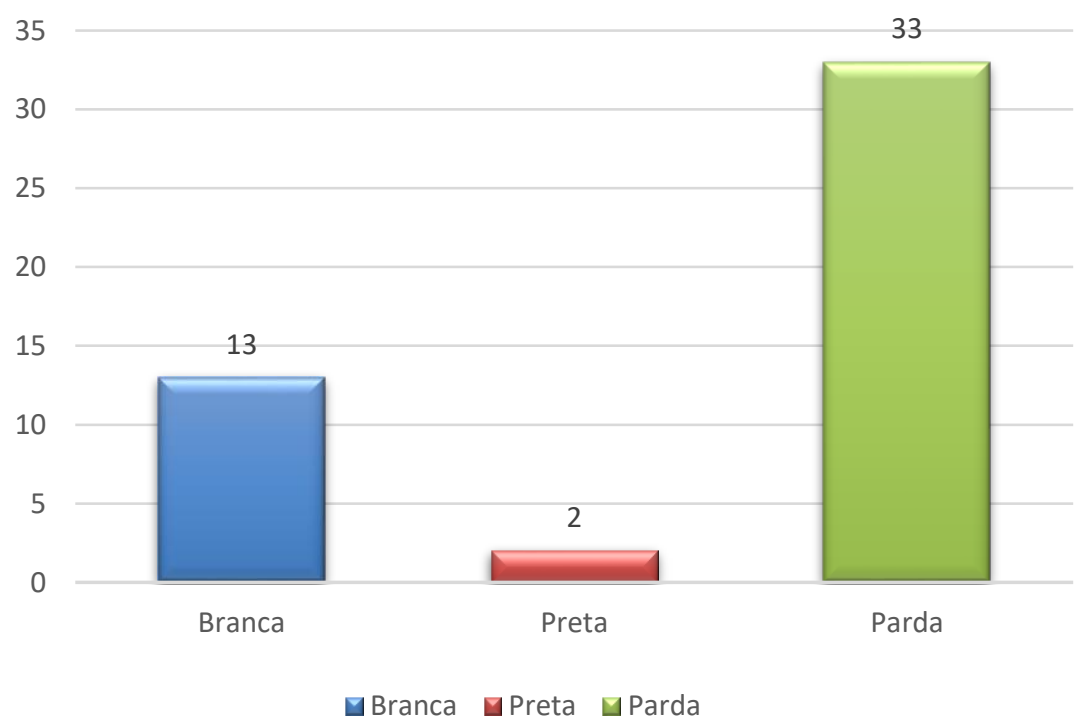


Figura 5: Casos confirmados por raça.

Em relação a escolaridade, $91,66 \%$ dos casos notificados não tiveram especificados a escolaridade dos pacientes na hora da notificação, ou simplesmente foram ignorados (demonstrado na figura 6). É de extrema importância a correta notificação dessa variável uma vez que as instruções de educação em saúde devem ser repassadas ao paciente em uma linguagem clara e concisa, de forma que ele possa entender corretamente a mensagem repassada.

Sabe-se que indivíduos com baixa condição socioeconômica moram em regiões de risco ou locais periféricos com precariedade no sistema sanitário, ou morando em locais de encostas, próximo a matas etc. Isso os expõe com maior facilidade ao agente causador da DC. Soma-se a isso o fato de maior acometimento no segundo semestre, os vetores saem dos seus locais de habitat (devido a queimadas) e rumam ao peridomicílio, colocando em risco essa população.

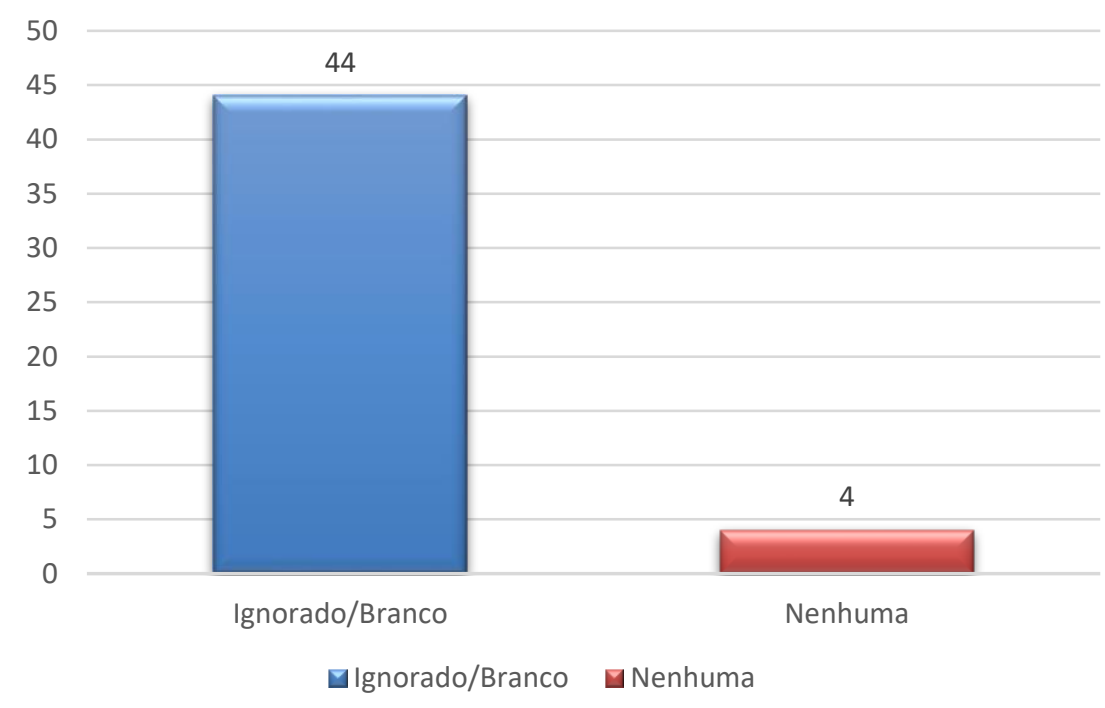

Figura 6: Casos confirmados por escolaridade.

A grande maioria dos casos notificados foram notificados como sendo de origem na zona urbana $(79,16 \%$ dos casos). Isso demonstra que atualmente ocorre uma inversão do perfil de acometimento que passa a ser mais centralizado na zona urbana e de forma que acomete casos isolados ou surtos familiares através do consumo de alimentos in natura que são comuns na região do Tocantins.

Outro ponto importante para entender esse novo perfil de acometimento é a existência de ocupação de lugares periféricos nas cidades (como citado anteriormente) e 
isso implica diretamente que pessoas com baixa condição econômica ocupam essas localidades ambientalmente fragilizadas e com ocorrência da doença nessas localidades, seja por meio oral ou vetorial. Isso corrobora com o fato de atualmente existir uma inversão no perfil epidemiológico da DCA, em que outrora ocorria mais em ambientes silvestres, e nos dias de hoje ocorre uma urbanização da doença.

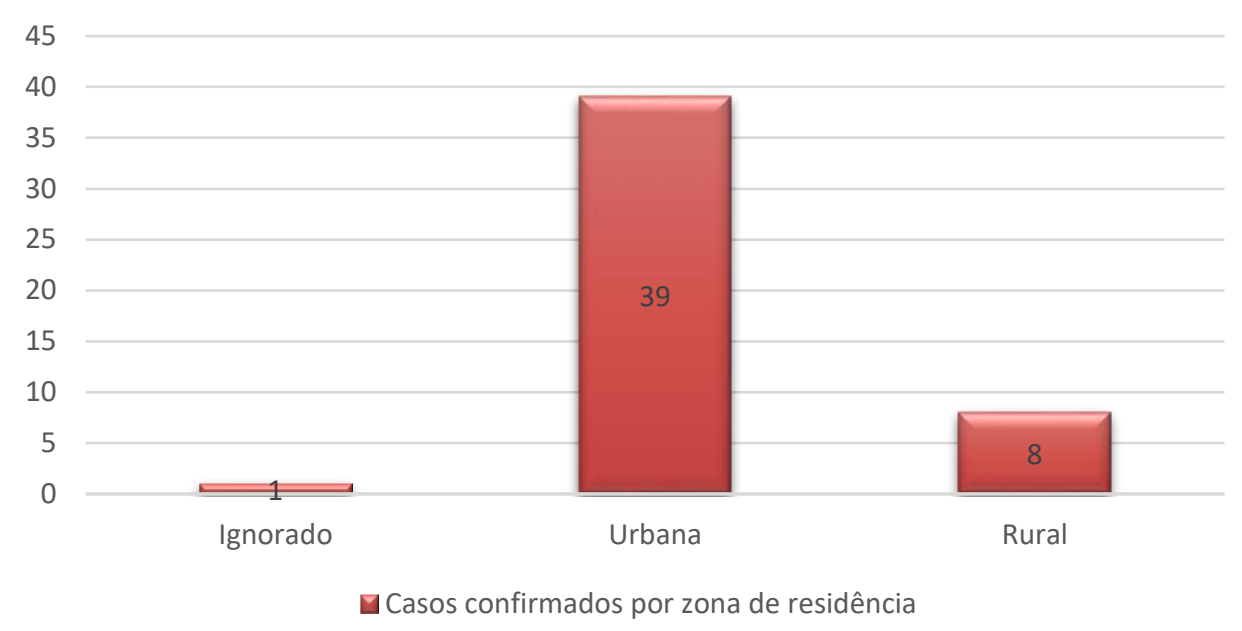

Figura 7: Casos confirmados por Zona de Residência.

Segundo Ferreira, Branquinho e Leite (2014), até meados dos anos 2000 os casos de DCA oriundo da transmissão oral era um evento pouco visado e, por esse motivo, pouco investigado na época. Atualmente tornou-se frequente na região amazônica e está relacionada à ocorrência de surtos recentes em diversos estados brasileiros.

Ainda segunda Ferreira, Branquinho e Leite (2014) a primeira notificação de DCA por infecção oral foi registrado por Mazza (Argentina) no ano de 1936, através da ingestão do leite materno.

Analisando à provável fonte de transmissão, se oral ou vetorial (conforme a figura 7), $77,08 \%$ dos casos ocorreram com transmissão oral. Isso se deve ao fato de que no estado do Tocantins há o consumo tradicional do açaí, bacaba e outras frutas de modo in natura. A forma vetorial foi responsável por apenas $10,41 \%$ dos casos de DCA. Isso também demonstra uma inversão do tipo de infecção em que outrora era de forma mais expressiva pela forma vetorial. 


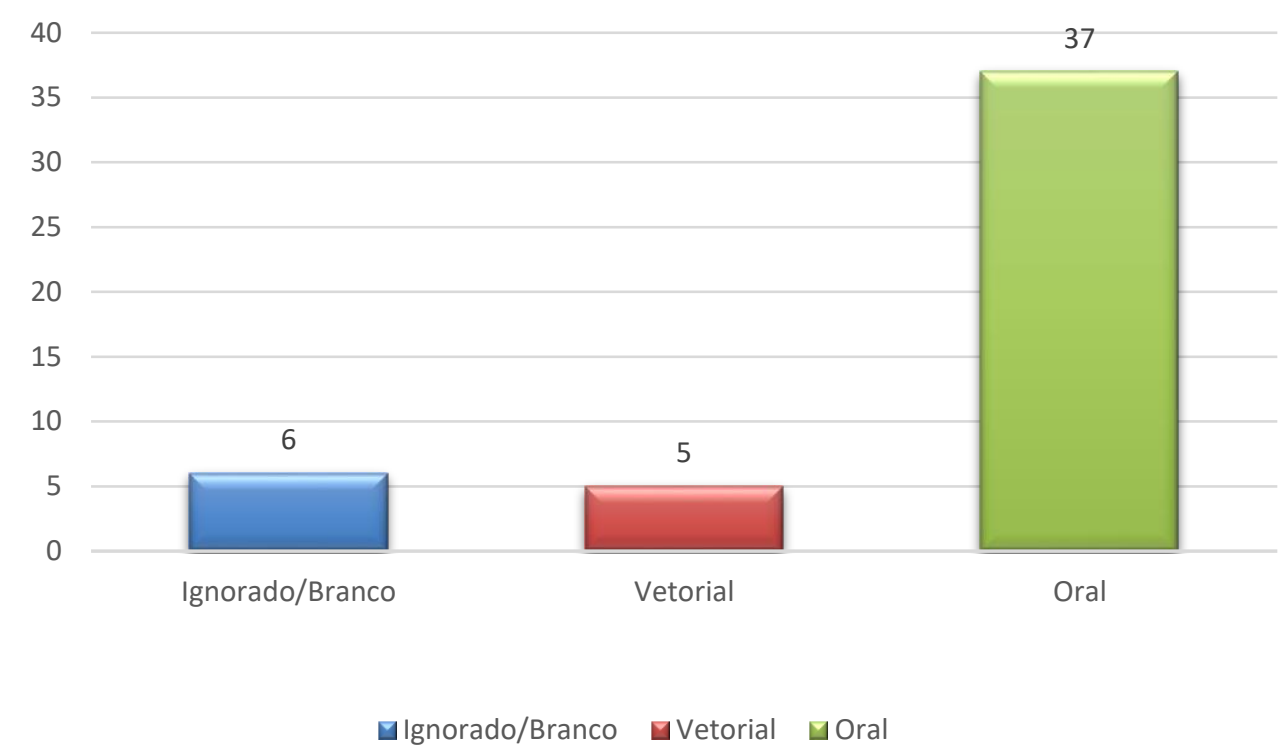

Figura 8: Casos confirmados por modo provável de infecção.

\section{CONSIDERACOOES FINAIS}

Durante a realização desta pesquisa observou-se que existem diversas relações epidemiológicas associadas a notificação de DCA no Estado do Tocantins, como por exemplo, variáveis epidemiológicas, ambientais e demográficas, no período de 2007 a 2018.

Foram notificados 48 casos confirmados de DCA, com predominância da doença entre mulheres, com idade entre 15-39 anos. Do total, 77,08\% tiveram como modo provável de infecção a transmissão oral. Observou-se também a ocorrência de maior número de casos no segundo semestre dos anos analisados. Isso se deve ao fato de que é nesse período que ocorrem as altas temperaturas associadas a baixos índices pluviométricos. Isso ocasiona uma maior ocorrência de queimadas, provocando a saída do vetor de seu habitat natural que migra ao peridomicílio, entrando em contato com os seres humanos.

Outro fato relacionado a maior ocorrência de casos no segundo semestre é a maior produção de açaí (produto muito consumido no Estado do Tocantins). Com maior produção existe uma maior disponibilidade e maior probabilidade de contágio.

Existe uma grande incidência de DCA nos últimos anos no Estado do Tocantins mesmo apesar da sintomatologia na fase aguda da doença geralmente ser pouco evidente, o que pode levar a subnotificação da doença. 
Dessa forma concluímos que o perfil epidemiológico dos pacientes estudados é um indivíduo adulto, do sexo feminino, raça parda, escolaridade não informada em grande parte das notificações, morador da zona urbana, com infecção de modo oral e em domicílio. A descrição do perfil epidemiológico é de suma importância para gerar informações e traçar medidas de educação em saúde, combate ao vetor e de correto atendimento e entendimento dos sinais e sintomas para prevenir a doença e aumentar o diagnóstico precoce.

Mesmo existindo muitas estratégias sendo implementadas no combate à doença de Chagas transmitida por via oral, ainda existe a necessidade de mais estudos, pesquisas e incentivo para que os profissionais que atuam na atenção à saúde tenham a correta compreensão dos sinais e sintomas, para realizar o pronto atendimento desses pacientes atingidos pela forma aguda da doença de Chagas. A educação em saúde é de suma importância para ensinar boas práticas de higiene e consumo de alimentos naturais, contribuindo para evitar que eventos assim ocorram.

\section{REFERÉNCIAS}

ALMEIDA, P. J. DE et al. Relação entre os casos da doença de Chagas e a produção de açaí no norte do Brasil. Sociedade Brasileira de Medicina Tropical, v. 2, p. 0, 2016.

COSTA, F. D. A. et al. O eletrocardiograma na fase aguda da Doença de Chagas por transmissão oral. Revista Brasileira de Cardiologia, p. 127-130, 2013.

FERREIRA, R. T. B.; BRANQUINHO, M. R.; LEITE, P. C. Transmissão oral da doença de Chagas pelo consumo de açaí: um desafio para a Vigilância Sanitária. Vigilância Sanitária em Debate, v. 2, n. 4, p. 4-11, 2014.

FLÁVIA, A. et al. Doença de Chagas e a transmissão por alimentos contaminados Dysfunctions due to schistosomiasis. Revista Brasileira de Educação e Saúde, p. 130135, 2020.

LOYANE, G.; CARVALHO, B. Doença e Chagas: Sua transmissão através do consumo de açaí. Acta de Ciências e Saúde, v. 01, n. 01, p. 24-36, 2018.

ORTIZ, J. V. et al. Cardiac evaluation in the acute phase of chagas' disease with posttreatment evolution in patients attended in the state of Amazonas, Brazil. Arquivos Brasileiros de Cardiologia, v. 112, n. 3, p. 240-246, 2019.

PINTO, A. Y. DAS N. et al. Doença de chagas aguda grave autócne da Amazônia brasileira. Revista Paraense de Medicina, v. 21, n. 2, p. 1-10, 2010. 
RASSI, A.; RASSI, A. Doença De Chagas Aguda. Revista de Patologia Tropical, v. 36, n. 3, 2010.

RIBEIRO, S. A. et al. Análise e perspectiva do controle da doença de Chagas no Brasil. Revista brasileira de malariologia e doenças tropicais. Publicações avulsas, v. 17, n. 1, p. 387-402, 2017.

SANGENIS, L. H. C. et al. Transmissão da doença de Chagas por consumo de carne de caça: revisão sistemática. Revista Brasileira de Epidemiologia, v. 19, n. 4, p. 803-811, 2017.

SANTOS, J. A. C. DOS; PEREIRA, F. C. DA S. Epidemiologia da Doença de Chagas no Estado do Amapá no período de 2010 a 2013. Journal of Chemical Information and Modeling, v. 53, n. 9, p. 1689-1699, 2013.

SHAW, J.; LAINSON, R.; FRAIHA, H. Considerações sobre a epidemiologia dos primeiros casos autóctones de doença de Chagas registrados em Belém, Pará, Brasil. Revista de Saúde Pública, v. 3, n. 2, p. 153-157, 1969.

TATTO, E.; PADILHA, E. MAURA; FERNANDES, S. B. Doença de Chagas Aguda: Aspectos epidemiológicos, diagnóstico e tratamento. Guia de consulta rápida para profissionais de saúde. Programa nacional de Controle de Chagas, Secretaria de Vigilância em Saúde, Ministério da Saúde., p. 32, 2007.

VARGAS, A. et al. Investigação de surto de doença de Chagas aguda na região extraamazônica, Rio Grande do Norte, Brasil, 2016. Cadernos de Saúde Pública, v. 34, n. 1, 2018.

WANDERLEY, D. M. V. et al. Doença de Chagas: a vigilância entomológica no Estado de São Paulo. Boletim Epidemiológico Paulista, v. 4, p. 8-12, 2007. 